Forsberg, H., M. Jonsson, and B. Svensson, "Embedded signal processing using free-space optical hypercube interconnects," SPIE Optical Networks Magazine, vol. 4, no. 4, pp. 35-49, July/Aug. 2003.

\section{Embedded signal} processing using free-space optical hypercube interconnects

\author{
Håkan Forsherg
}

Department of Computer Engineering

Chalmers University of Technology

Göteborg, Sweden

\section{Magnus Jonsson}

\section{Bertil Suensson}

School of Information Science

Computer and Electrical Engineering

Halmstad University

Halmstad, Sweden
Copyright 2003 Society of Photo-Optical Instrumentation Engineers and Kluwer Academic Publishers. This paper was published in Optical Network Magazine and is made available as an electronic reprint with permission of SPIE and KAP. One print or electronic copy may be made for personal use only. Systematic or multiple reproduction, distribution to multiple locations via electronic or other means, duplication of any material in this paper for a fee or for commercial purposes, or modification of the content of the paper are prohibited.

\section{ABSTRACT}

The speed and complexity of integrated circuits are increasing rapidly. For instance, today's mainstream processors have already surpassed gigahertz global clock frequencies on-chip. As a consequence, many algorithms proposed for applications in embedded signal-processing (ESP) systems, e.g. radar and sonar systems, can be implemented with a reasonable number (less than 1000) of processors, at least in terms of computational power. An extreme inter-processor network is required, however, to implement those algorithms. The demands are such that completely new interconnection architectures must be considered.

In the search for new architectures, developers of parallel computer systems can actually take advantage of optical interconnects. The main reason for introducing optics from a system point of view is the strength in using benefits that enable new architecture concepts, e.g. free-space propagation and easy fan-out, together with benefits that can actually be exploited by simply replacing the electrical links with optical ones without changing the architecture, e.g. high bandwidth and complete galvanic isolation.

In this paper, we propose a system suitable for embedded signal processing with extreme performance demands. The system consists of several computational modules that work independently and send data simultaneously in order to achieve high throughput. Each computational module is composed of multiple processors connected in a hypercube topology to meet scalability and high bisection bandwidth requirements. Free-space optical interconnects and planar packaging technology make it possible to arrange the hypercubes as planes with an associated three-dimensional communication space and to take advantage of many optical properties. For instance, optical fan-out reduces hardware cost. Altogether, this makes the system capable of meeting high performance demands in, for example, massively parallel signal processing. One 64-channel airborne radar system with nine computational modules and a sustained computational speed of more than 1.6 Tera floating point operations per second (TFLOPS) is presented. The effective inter-module bandwidth in this configuration is $1024 \mathrm{Gbit} / \mathrm{s}$. 


\section{Introduction}

A lgorithms recently proposed for applications in embedded signal-processing (ESP) systems, e.g. in radar and sonar systems, demand sustained performance in the range of 1 GFLOPS to 50 TFLOPS [1]. As a consequence, several processors must work together, thus increasing inter-processor communication. Moreover, the data transfer time increases quickly if an incorrect network topology is chosen, especially in systems with frequent use of all-to-all communication structures. The choice of a scalable high-speed network is therefore essential. Other requirements that must be fulfilled in ESP systems are real-time processing, low power consumption, small physical size, and multimode operation. New parallel computer architectures are necessary to be able to cope with all these constraints at the same time.

Several parallel and distributed computer systems of this kind for embedded real-time applications have been proposed in the literature, including systems that make use of fiber optics in the interconnection network in order to achieve high bandwidth. See, for instance, Jonsson $[3,15]$. However, to make the best use of optics in interprocessing computing systems, all properties of optics and optoelectronics must be taken into consideration. Among these properties is the ability to communicate in free-space in all spatial dimensions as well as, e.g., high fan-out [5].

In fact, a careful evaluation of optics in general shows that both properties that enable new architecture concepts and properties that can be exploited without changing the architecture increase system performance. In other words, optics not only provides, e.g., higher bandwidth and complete galvanic isolation, it also provides a new design space. This means in reality that optical technologies can improve some important properties of ESP systems. For instance, optics can reduce the physical size and improve the bandwidth over the cross section that divides a network into two halves, usually referred to as the bisection bandwidth (BB) [4]. High BB reduces the time it takes to redistribute data between computational modules that process information in different dimensions. This reduction of time is very important in ESP systems [4].

It has furthermore been shown that optical freespace interconnected 3D systems (systems using all three spatial dimensions for communication), with globally and regularly interconnected nodes arrayed on planes, are best suited for parallel computer architectures using optics [7-9]. Folding optically connected 3D systems into planes also offers precise alignment, mechanical robustness and temperature stability at a relatively low cost [10].

In this paper, we propose a system consisting of several clusters of free-space optically interconnected processing elements. The clusters are linked together in a pipeline fashion to attain high throughput and to meet the pipelined data flow nature of ESP systems. The processing elements within a cluster are globally and regularly connected in a binary hypercube topology and are finally transformed into a plane. Note, however, that the use of optics does not allow one to collapse higher dimensional graphs to lower dimensional graphs, since even free-space optics requires a minimum finite crosssectional area. What occurs is that this cross-sectional area is sometimes so small that it no longer limits the implementation, and other factors such as the size of the elements then become limiting factors.

Other networks that have employed the positive features of the hypercube in combination with optics are, e.g., the Spanning Multichannel Linked Hypercube (SMLH) network [18], the Optical Transpose Interconnection System (OTIS) hypercube [19] and the GlobalLocal Optical Reconfigurable Interconnect (GLORI) network [20].

Our new hardware architecture is evaluated with a 64-channel airborne space-time adaptive processing (STAP) radar application. The sustained computational load is more than 1.6 TFLOPS. As a consequence, several hundreds of processors must be used to reduce the per-processor load. In addition, certain all-to-all communication structures must also be used in the signal processing chain. This altogether evaluates the performance of the inter-processor communication network in the new architecture.

Figure 1 depicts an example of the proposed system architecture. Note, however, that the intra-cluster hypercube formation is not shown here but later in the text.

The paper is organized as follows. Section 2 introduces the binary hypercube topology and explains how some important all-to-all communication can be implemented on it. In Section 3, we describe how it is possible to merge interconnection topologies, including the hypercube, into optical planes. In Section 4, we show how it is possible to massively interconnect these optical planes using free-space optics and describe the advantages of doing so. The case study discussed in Section 5 demonstrates the power of using pipelined optical hypercubes in embedded signal processing systems. The paper is concluded in Section 6.

\section{Hypercubes}

The binary hypercube (hereafter called simply the hypercube) is a flexible topology with many attractive features. For instance, several well-known topologies, such as meshes, butterflies and shuffle-exchange networks, can be embedded into it [12]. Rings with an even number of nodes and certain balanced trees can also be embedded [2]. Another feature that makes the hypercube attractive is that the bisection bandwidth (BB) scales linearly with the number of processors, and thus higher dimensions lead to very high $\mathrm{BB}$. High $\mathrm{BB}$, as indicated in the introduction, is of great importance in ESP systems.

Geometrically, a hypercube can be defined recursively as follows: The zero-dimensional hypercube is a single processor. An $n$-dimensional hypercube with $N=2^{n}$ PEs 


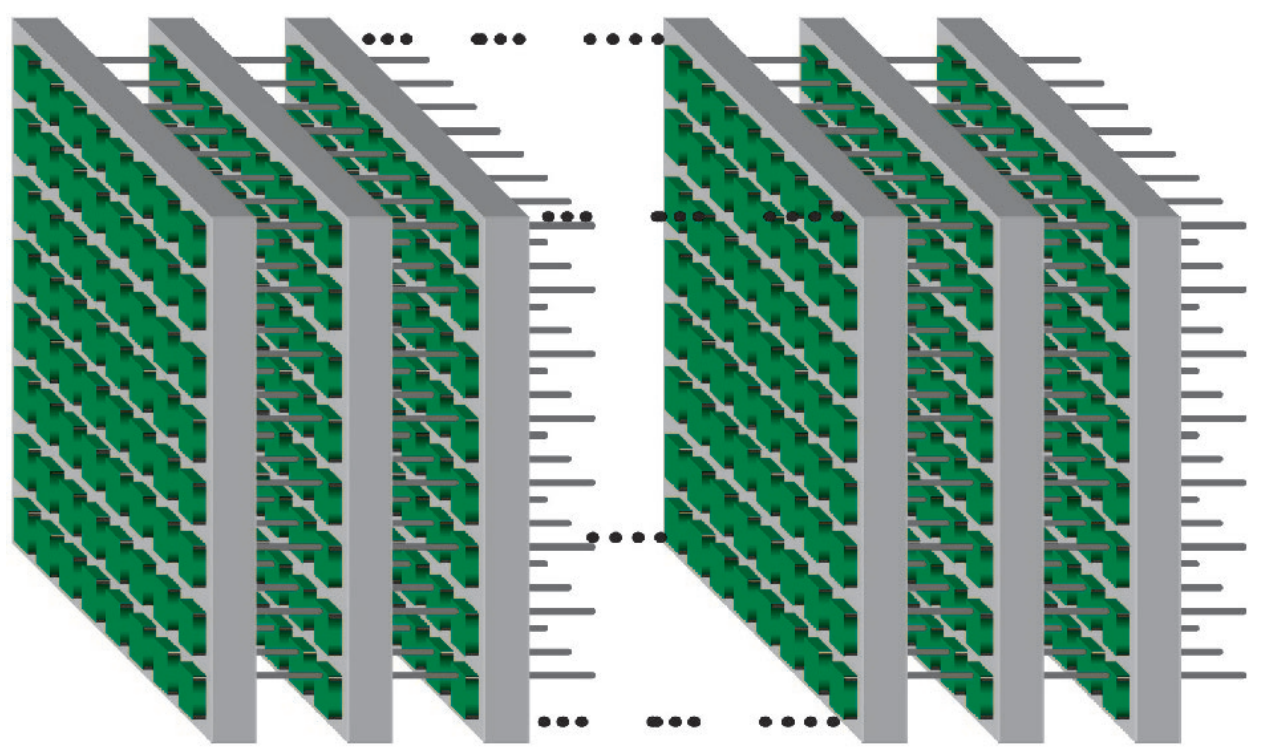

Figure 1: Several 6D hypercubes transformed into planes and massively interconnected-an example of the proposed system architecture.

is built of two hypercubes with $2^{n-1}$ PEs, where all the PEs in one half are connected to the corresponding PEs in the other half. See Figure 2, which shows a six-dimensional hypercube (Figure 2c). This hypercube is built of two 5D hypercubes, which in turn are built of $4 \mathrm{D}$ hypercubes (Figure 2b). The 4D hypercube is further subdivided into 3D hypercubes (Figure 2a). Note that the thick lines in Figure 2c consist of eight interconnections each.

A disadvantage of the hypercube is its complexity. It requires more and longer wires than the mesh, since not only the nearest physical neighbors are connected to each other if the dimension is greater than three, i.e. more dimensions than in physical space [13]. The fact is that the amount of electrical wires already required (of different lengths) in a relatively small hypercube will be enormous. Consider, for instance, an implementation of a 6D hypercube on a printed circuit board, where the transfer rate of a unidirectional channel between two processing elements must be in the order of $10 \mathrm{Gbit} / \mathrm{s}$. This implementation requires 12288 electrical wires of different lengths, each clocked with a frequency of 312.5 $\mathrm{MHz}$ (assuming 32-bit wide channels). Since the wires are of course not allowed to cross each other physically, many layers will be required.

\subsection{Communication in hypercubes}

Hypercubes can be used to implement several algorithms requiring all-to-all communication, e.g. matrix transposition, vector reduction, and sorting [13]. It is also easy to implement broadcasting in this architecture [6]. Two of the global communication structures, corner a)

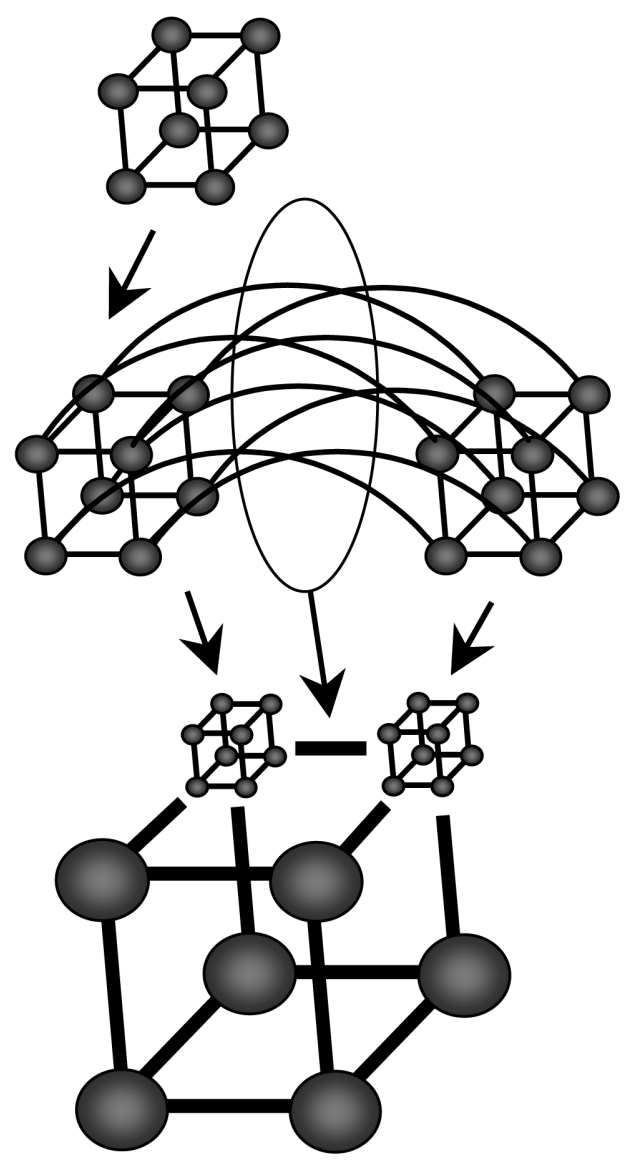

Figure 2: a) 3D hypercube. b) 4D hypercube built of two $3 \mathrm{D}$ hypercubes. c) 6D hypercube built of two $5 \mathrm{D}$ hypercubes, which in turn are built of $4 \mathrm{D}$ hypercubes. 
turning and broadcasting, are of specific importance in embedded signal processing, and we thus discuss them in detail here. (Note that the corner turn algorithm is the same as a matrix transposition from a mathematical point of view [4].)

- Corner Turning

In corner turning, all nodes send a unique data set to all other nodes. Corner turning is used to efficiently redistribute data between pipeline stages that process information in different dimensions. The following example is given to illustrate this:

Assume that we have collected 640 samples in total from eight radar channels (RCs), i.e. 80 samples per channel. Further, assume that these samples are distributed channel-wise over eight processing elements (marked 1-8 in Figure 3). Samples from RC 1 (labeled in Figure 3a) are located in processing element (PE) 1, samples from RC 2 are located in PE 2 etc. In the subsequent calculation step (the next pipeline stage), samples 1-10 from all RCs must be processed in PE 1, samples 11-20 from all RCs must be processed in PE 2 etc. This means that samples 1-10 from PE 2-8 must be redistributed to PE 1, samples 11-20 from PE 1 and PE 3-8 must be redistributed to PE 2 etc. The final result is shown in Figure $3 \mathrm{~b}$. Here, one can see that the original data from PE 1 has been scattered over all PEs. The same thing has occurred for all other PEs' original data. This process (the process that takes place between Figure $3 \mathrm{a}$ and $3 \mathrm{~b}$ ) is called corner turning.

One way to efficiently implement corner turning in the hypercube is to use the hypercube transpose algorithm described by Foster [13]. This algorithm is particularly good when transfer costs are low and message start-ups are expensive [13]. Therefore, optical interconnects with their slightly higher start-up cost and high bandwidth typically match the transpose algorithm behavior better than pure electrical wires. Furthermore, the hypercube transpose algorithm, as well as the broadcasting algorithm described below, only transfer data in such way that cost-saving optical beam splitters can be used in the interconnection network.
In the hypercube transpose algorithm, half of the chunk of data to be redistributed is exchanged in every dimension. Assume that $D_{\text {size }}$ is the total size of the chunk of data in number of bits, $P$ is the number of processors in the hypercube and $R_{\text {link,eff }}$ is the efficient transfer rate in bits per second of a single link in one direction when overhead is excluded, e.g. message start-up time. Then, a full corner turn takes:

$$
\frac{\frac{1}{2} D_{\text {size }} \log _{2}(P)}{P R_{\text {link, eff }}}
$$

seconds. Note that $\log _{2}(P)$ corresponds to the number of dimensions in the hypercube and that product $P R_{\text {link,eff }}$ corresponds to the bisection bandwidth (BB). Thus, the reorganization time is proportional to the product of the data chunk size and the cube dimension divided by the BB.

Using this one-dimension-at-a-time procedure means that we can make use of cost-saving single ports, i.e. we use optical beam splitters to reduce the number of transmitters. Note also that beam splitters allow each node to transmit the same data to more than one neighbor at the same time. This is an extra feature compared to genuine single-port communication, where a node can only send and receive on one of its ports at the same time. On the other hand, transmitting the same data set to more than one node is the exact opposite to corner turning, where each node sends a unique data set to all other nodes.

- Broadcasting

In broadcasting, all nodes need to copy information from themselves to all other nodes or a subset thereof. Broadcasting can, for instance, be used in the constant false alarm ratio (CFAR) stage in certain radar algorithms [5]. CFAR is however not considered in this article.

As in the transpose algorithm described above for hypercubes with one-port communication, the data transfer time for broadcasting is minimized if one dimension is routed at a time. Using this principle, each

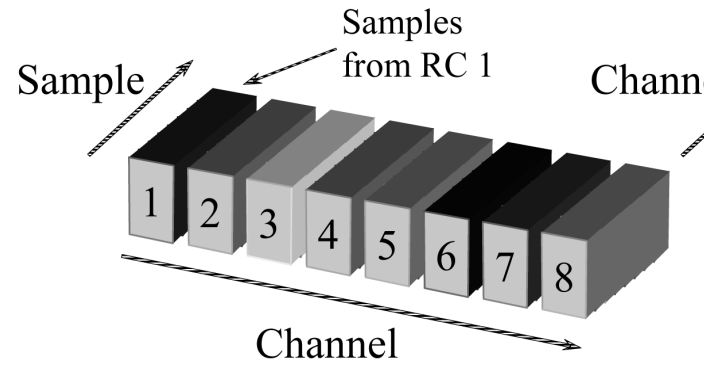

a)

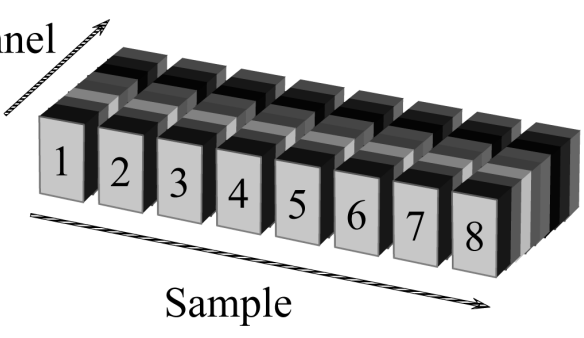

b)

Figure 3: The corner turning process, i.e. the process of redistributing data between pipeline stages that compute in different dimensions. 
node copies its own amount, $M$, of data to its first neighbor (along the first dimension), and simultaneously receives the same amount of data from the same neighbor. The next time, each node copies its own data, and the data just received from the first neighbor, to the second neighbor (along the second dimension), and simultaneously receives the same amount (i.e. $2 M$ ) of data. This procedure is repeated over all dimensions in the hypercube. Thus each node has to send and receive the following amount of data:

$$
\sum_{i=0}^{\log _{2}(P)-1} 2^{i} M=(P-1) M
$$

where $M$ is the data size (in number of bits) in each node that must be copied to all other nodes in the hypercube and $P$ is the number of processors (nodes). Again, assuming that each node has an efficient transfer rate of $R_{\text {link, eff }}$ bits per second, broadcasting will take:

$$
\frac{(P-1) M}{R_{\text {link,eff }}}
$$

seconds. Note, however, that this expression is valid only if we consider the nodes as single port. In reality, as described above, using optical technology, a copy of data from one node can actually be distributed to all $\log _{2}(P)$ neighbors at the same time and each node can in fact receive data from all its neighbors at the same time. Of course, this is true only if (i) we are sure that we do not exceed the optical fan-out limitation of the given technology (here, planar free-space optics), (ii) the receivers are capable of detecting the weaker signals that result from the optical beam splitting and (iii) the receiving nodes are capable of processing multiple information flows at the same time at the given speed. If all this holds, the time it takes to broadcast data can be reduced to:

$$
\frac{\left\lceil\frac{(P-1)}{\log _{2}(P)}\right\rceil M}{R_{\text {link,eff }}}
$$

seconds. To illustrate this, assume that, in a first phase, a single node receives data from all its $\log _{2}(P)$ neighbors simultaneously. Then, in a second phase, the same node receives data originating from its neighbors' neighbors, i.e. the data that were received by the neighbors in the first phase. The data transfer is then completed when the node has received data from all $(P-1)$ nodes. Note, however, that, at the most, $\log _{2}(P)$ neighbors, i.e. the number of physical links, can deliver data at the same time to the same node.

To conclude, it seems that the hypercube topology with its high bisection bandwidth is a good candidate for
ESP systems that frequently perform corner turning. On the other hand, the hypercube is still afflicted with its high interconnection complexity. However, restricting the nodes to only sending data with one transmitter each can reduce this complexity. In addition, if optical beam splitters and multiple receivers are used together with single transmitters, the broadcast capabilities of the network can be enhanced. Finally, as will be shown in next section, free-space optics and optical planar packaging technology even further reduce the interconnection complexity in hypercubes.

\section{Hypercubes in Planar Free-Space Optics}

There are many reasons to fold optically interconnected 3D systems into planes. One reason, as will be demonstrated in this section, is that complicated network topologies can be transformed into these planes of nodes with associated three-dimensional communication space. (Remember from the introduction that optics does not collapse higher dimensional graphs to lower, but reduces the cross-sectional area to a size and form where it no longer limits the implementation.) Other reasons to fold optically interconnected systems into planes are precise alignment, mechanical robustness and the ease of cooling, testing and repairing the optoelectronic and electronic circuits attached to the substrates [10,11].

In free-space optical planar technology, waveguides are made of glass or transparent semiconductor-based substrates. These substrates serve as a light traveling medium as well as a motherboard for surface mounted optoelectronic and electronic chips [11]. Micro-optical elements such as beam splitters and microlenses can also be attached to both sides of the substrate. To be able to enclose the optical beams in the light traveling medium, the surfaces are covered with a reflective structure. The beams will hence "bounce" on the surface.

In the following three steps, 1-3, and Figures, 4-6, we show how a $3 \mathrm{D}$ hypercube topology is merged into

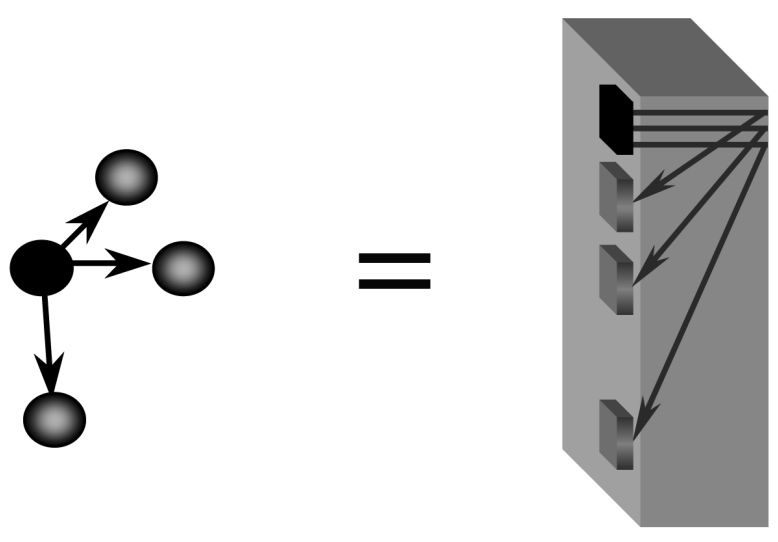

Figure 4: Topological and physical view over four PEs in a 3D hypercube, where the black PE sends data to its three neighbors. 

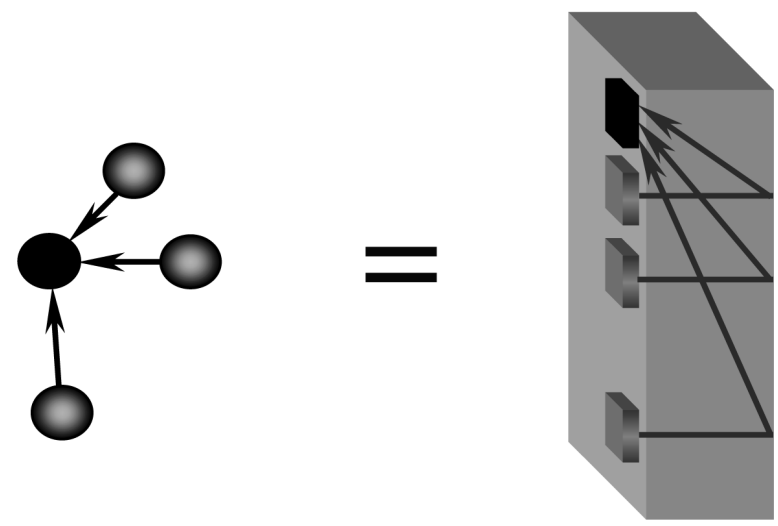

Figure 5: Topological and physical view of four PEs in a $3 \mathrm{D}$ hypercube, where the black PE receives data from its three neighbors.
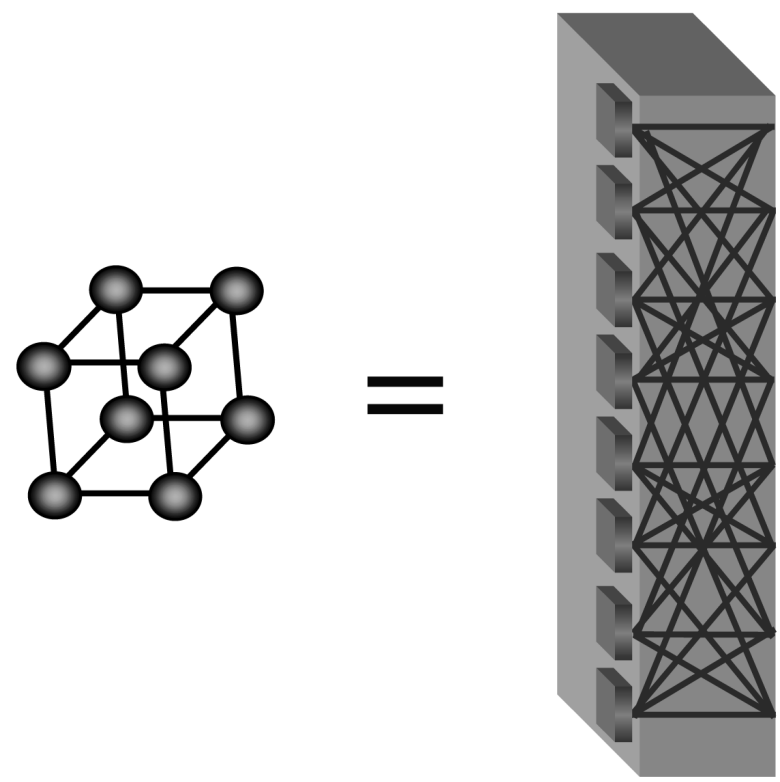

Figure 6: All transmitters and receivers in a row form a $3 \mathrm{D}$ hypercube. a free-space optical plane. In step 4 and Figure 7, we show how two 3D hypercubes can be combined into a $4 \mathrm{D}$ hypercube. In step 5 and Figure 8, we show how a complete 6D hypercube is merged into a free-space optical plane. Finally, in step 6 and Figure 9, we show how beam splitters can be used to reduce the number of transmitters or to enhance the interconnection network.

Before we start with step one, however, it must be clarified that it is possible to implement higher dimensional hypercubes (higher than 6D) on a single substrate in the same way as shown below (provided that it is physically possible). The choice of a 6D hypercube is only for the purpose of illustration. The placement of the processing elements is also chosen to be as illustrative as possible and is thus not to be considered as the only one. Finally, many other topologies can be merged in the same way as the hypercube. Observe, however, that rings, meshes, butterflies and shuffle-exchange networks are automatically merged into the substrate when the hypercube is merged, since these topologies are by default embedded into the hypercube. See Section 2.

- Step 1: Transmitters

In a $3 \mathrm{D}$ hypercube, each processing element has three neighbors. In Figure 4, it is shown, both topologically and physically, how one PE, colored black, sends data to its three neighbors.

- Step 2: Receivers

In the same way, a PE must be able to receive data from its three neighbors, see Figure 5.

- Step 3: Complete 3D hypercubes

In Figure 6, all transmitters and receivers in one row have been added. This corresponds topologically to a 3D hypercube.

- Step 4: 4D hypercubes

To create a 4D hypercube, we make use of two rows. See Figure 7. In the topological view in this figure, all PEs in the left 3D hypercube are connected to the nodes in the right hypercube. In the physical view, this corresponds
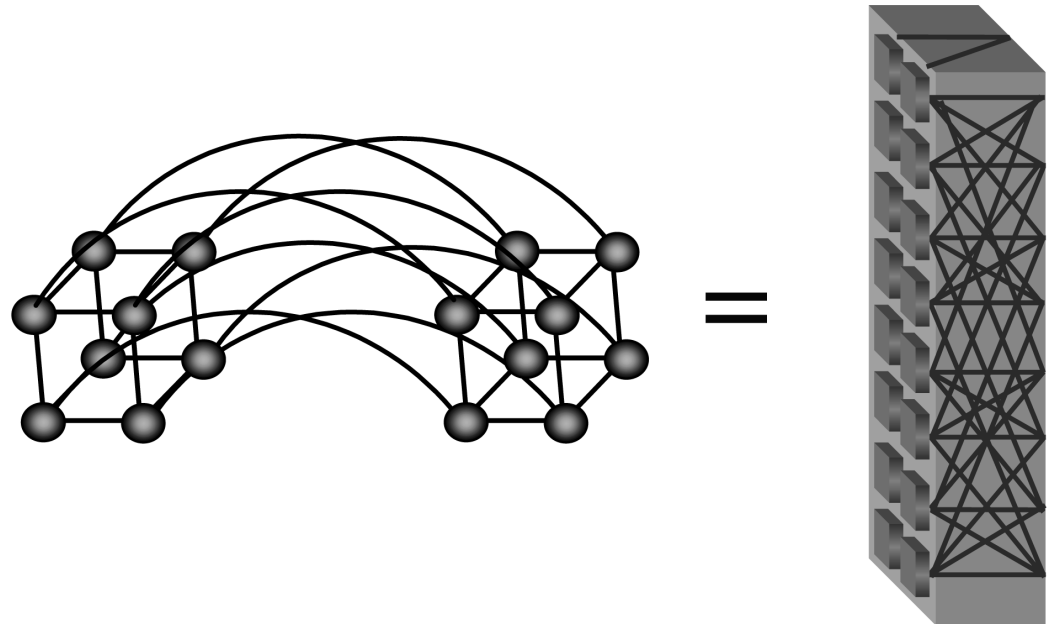

Figure 7: Two 3D hypercubes (rows) form a $4 \mathrm{D}$ hypercube. 

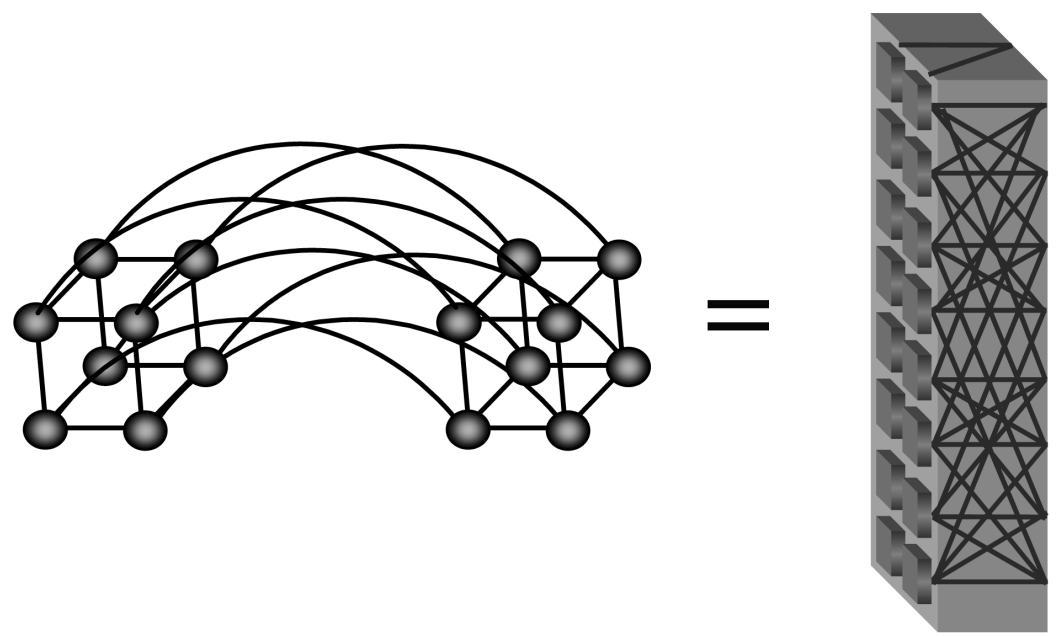

Figure 8: The whole computational module - a 6D hypercube.

to connections between each PE, and the corresponding one in the other. This is illustrated with a line on top of the substrate.

- Step 5: 6D hypercubes

A 6D hypercube makes full use of both horizontal and vertical space on a substrate (at least in this example), see Figure 8. The physical layout corresponds to a full computational module.

As mentioned earlier, micro-optical elements can be attached to the substrate. One such element is the optical beam splitter. If we use beam splitters in the hypercube, it is actually possible to reduce the number of transmitters by a factor equal to the number of dimensions without destroying the topology. As an example, if we want to implement a 6 D hypercube that originally has $384(6 \times 64)$ transmitters, it is sufficient to use 64 if we take full advantage of the beam splitters. The only restriction is that we must use some kind of channel time-sharing when different data must be sent to different neighbors at the same time, since only a single transmitter is available in each node. Figure 9 shows how optical beam splitters can be used to reduce the number of transmitters or to enhance the interconnection network.

Furthermore, if we use the hypercube transpose algorithm described by Foster [13] to perform corner turning, we will not lose any performance at all, even if we reduce the number of transmitters by a factor of six in a $6 \mathrm{D}$ hypercube as compared to a system without beam splitters. The trick in this algorithm is that data are exchanged in only one dimension at a time. Note, however, that the hypercube transpose algorithm sends more data and fewer messages in total, as compared to a simpler transposition algorithm also described by Foster [13]. Therefore, as mentioned above, the hypercube

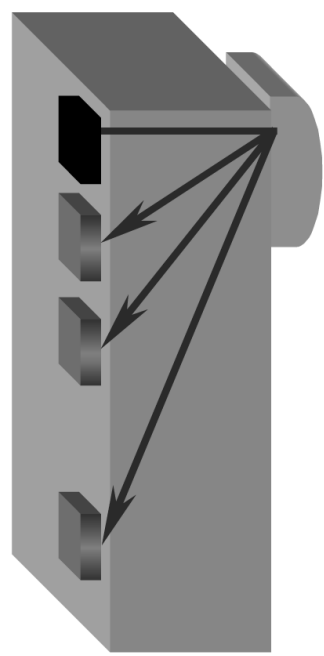

a)

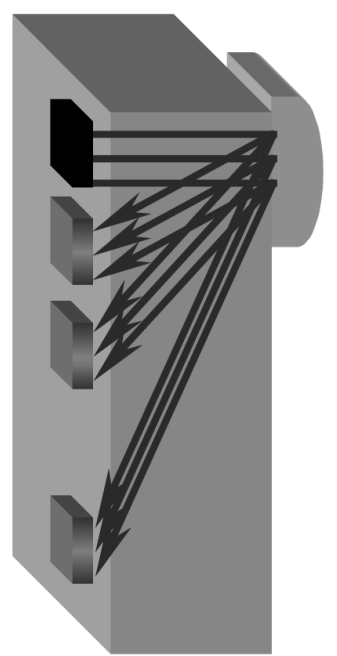

b)
Figure 9: a) Beam splitters are used to reduce the number of transmitters in a node (here by a factor of three). b) Beam splitters are used to increase the flexibility and multicast capacity in the network at the expense of more receivers but without additional transmitters.

transpose algorithm is preferable when transfer costs are low and message start-ups are expensive, e.g. in optical interconnection networks.

Last but not least, other sophisticated topologies can be created with beam splitters, although these will not be treated here.

- Step 6: Reduction of transmitters

As explained above, we will not lose any performance at all when we perform corner turning in the hypercube 
with fewer transmitters and with the help of beam splitters. As a consequence, we will use the beam splitters depicted in Figure 9a above. Note, however, that we split the light beam in both the horizontal and vertical directions, and thus maximally reduce the number of transmitters, i.e. by a factor of six.

In the same way as we investigated optical fan-out, we can analyze optical fan-in. In Figure 5, for instance, it is fully possible to use a single receiver for all beams. In that case, we must use some kind of time division multiple access to avoid data collisions and to synchronize all processing elements in the hypercube. However, with optical planar packaging technology, a synchronization clock channel is relatively easy to implement. Jahns [10], for instance, has described a 1-to-64 signal distribution suitable for, e.g., clock sharing, with planar packaging technology. However, since optical fan-in complicates our hypercubes with synchronization channels, we will put this issue aside.

\section{Pipelined Systems of Hypercubes}

If the required load exceeds the computational power in one module, several modules must co-operate. To make these modules work together efficiently, massively parallel interconnections are necessary. One way to interconnect several modules is to place them in succession, as in Figure 1. The possible drawback of this placement is that each plane can only send data forward and backward to the subsequent and the previous module, respectively. However, this arrangement suits the pipelined computational nature in most ESP systems and is therefore a good choice for such applications.

To make the inter-module communication work, we have to open up the substrates, i.e. let the light beams propagate via a lens from one module to the next. Figure 10 shows this inter-plane transmission. Specifically, Figure 10a shows the light propagation from one module to the next via a bottom surface lens. Figure 10b shows the top surface receiver. Note that it is possible to use a dedicated receiver module in Figure 10b instead of using the top surface of the computational unit as shown.

\subsection{D hypercube - two planar arrays}

The section above showed how the substrate is opened in order to connect computational modules. This procedure showed only how the modules were connected in one direction, however, i.e. one module could only receive from the previous module and send data to the next one. By allowing communication in both directions, i.e. letting a module be able to send and receive data both forward and backward, a 7D hypercube is actually formed by two planar arrays, see Figure 11 . If more than two planes form an extended computational module, the pure hypercube topology will not be preserved since only adjacent planes can communicate

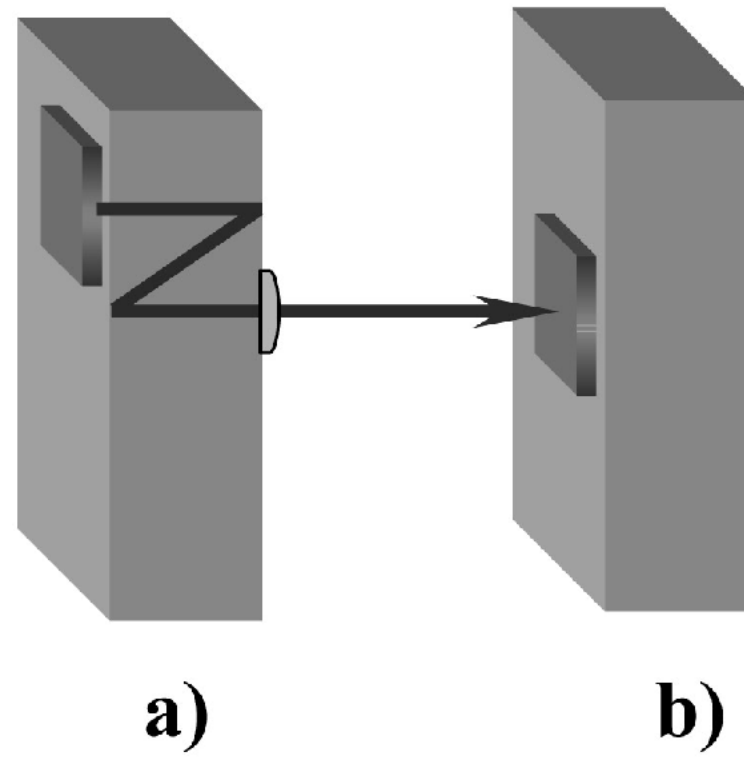

Figure 10: a) Inter-plane transmitting mechanism. b) Inter-plane receiving mechanism.

with each other. This, however, is not a limitation in a typical signal processing system owing to the pipelined nature of the data flow.

\subsection{Software scalability}

If only one mode of operation is needed in the system, we can create a streamed architecture for that purpose. However, since it is very important in many ESP applications to change the mode of operation on the same system as needed in the application, we would like to have an architecture capable of multimode operations. Thus, different clusters of computational units must be capable of working together in different ways.

The pipelined system described in this paper has very good potential for mapping of different algorithms in various ways. In fact, the system can be partitioned in all three spatial dimensions. An example of this is shown in Figure 12. In this picture, three different alternatives show exactly the same thing, namely, four different tasks mapped on four smaller systems of pipelined 4D hypercubes. It is also possible to create $5 \mathrm{D}$ hypercubes inside each of these four smaller systems by connecting two 4D hypercubes in different planes.

\subsection{Hardware scalability}

To be able to increase system performance in the future, hardware scalability is of great importance. Higher performance can be achieved in the proposed system by:

a) adding more planar arrays in the chain,

b) adding more PEs within a plane by denser packaging or

c) substituting a plane with more powerful PEs. 

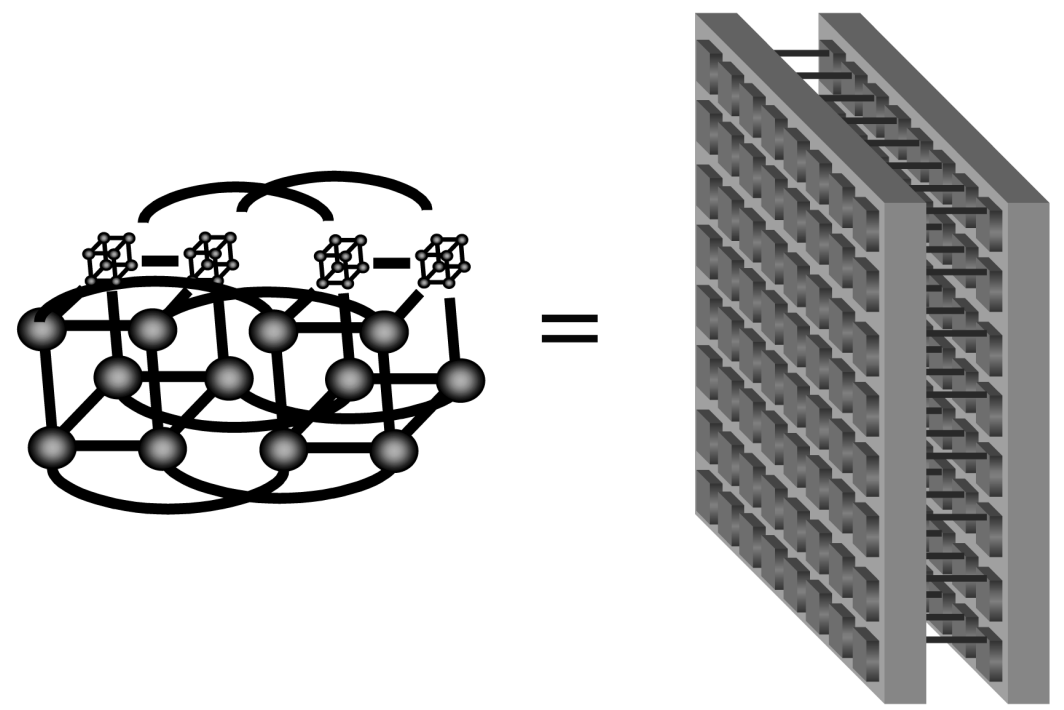

Figure 11: Topological and physical view of a $7 \mathrm{D}$ hypercube.

Note that, provided that it is possible to add more PEs within a plane, the intra-plane interconnection network will not be a bottleneck since the hypercube's bisection bandwidth scales well with the number of processors. However, to make it possible to add more processors within a plane, it must be noted that (i) at least twice as many PEs must be located on the same plane in order to preserve the pure hypercube technology (unless a more sophisticated inter-plane solution is used), (ii) either must the receivers be capable of detecting weaker signals if the optical beams are further subdivided as compared to the original solution or the transmitters must be more powerful and, finally, (iii) if all planes are not exchanged at the same time, the planes before and after the exchanged plane are not capable of receiving data from all PEs in the new plane. Furthermore, substituting a plane with more powerful PEs does not automatically mean that we enhance the network capacity. This may therefore lead to a processor/network imbalance. Apart from that, special attention must be paid to how the modules are stacked onto each other; e.g. heat removal etc. must be taken into account. All this being said, one should note that the addition of more planes is, generally speaking, facilitated by the fact that the inter-module links are free-space optical interconnects and that all modules are identical.
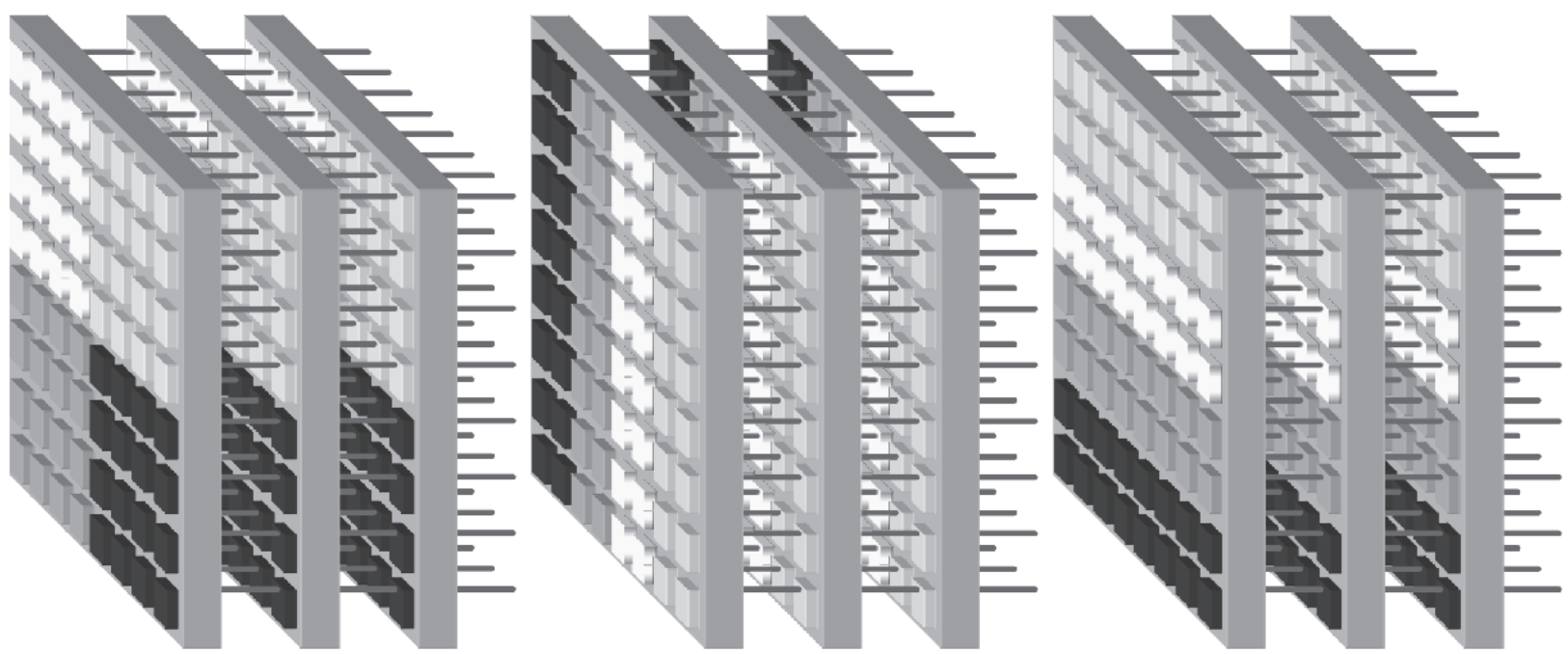

Figure 12: Three different alternatives of four independently working chains of $4 \mathrm{D}$ hypercubes. Each chain is marked with its own color. 


\section{Case Study}

An airborne radar signal processing unit for non-movable phased steered antennas is chosen as a case study. In this type of radar, it is possible to make adaptive beam forming in the signal processing chain and thus significantly improve the functional performance of the system. This process is often called space-time adaptive processing (STAP).

STAP requires a huge amount of calculations and puts high demands on inter-processor communication. Therefore, the new architecture must be capable of handling both high system load and high-volume interprocessor data transfers.

In this study, a single processing element is assumed to have a sustained floating point capacity of approximately 3 GFLOPS when all inter-processor data communication is excluded. In addition, for simplicity, no overlap between computation and communication is assumed.

\subsection{The airborne STAP-radar system}

STAP is an innovative tool for use with coherent phased array radar (and sonar) systems whenever the signals received are functions of both space and time [14]. STAP can, for instance, be used in airborne radar systems to support clutter and interference cancellation [17]. However, the full STAP algorithm is of little value in most applications since the computational workload is too high and it suffers from weak convergence [14]. Some kind of load-reducing and fast convergent algorithm is thus used. Examples are the nth order Dopplerfactored STAP, the medium (1st order) real-time STAP and the hard (3rd order) real-time STAP, all described by Cain et al. [17]. This study uses the same type of realtime STAP, although the computational load is increased many times. The reasons for this are, besides the higher order STAP (5th order), also the increase in input processing channels and the higher sampling rate etc.

The following system parameters are assumed for the airborne radar system:

- 64 processing channels $(L)$

- 5th order Doppler-factored STAP $(Q)$

- $32.25 \mathrm{~ms}$ integration interval (INTI) $(\tau)$
- 960 samples (range bins) $\left(N_{d}\right)$ per pulse after decimation with a factor of four

- 64 pulses per INTI and channel $\left(C_{p}\right)$

- $8 \mathrm{Gbit} / \mathrm{s}$ efficient data transfer rate of a single link in one direction $\left(R_{\text {link,eff }}\right)$

Because of the real-time nature of the system, a solution must have low computational latency. We thus put up a latency requirement slightly less than $100 \mathrm{~ms}$, i.e. a maximum time of $3 \tau$ to perform all calculations in the STAP chain from the input stage to the final stage. Note also that it is important to use as much time as possible without violating the latency requirement since the maximum latency determines the number of operations performed per time unit.

Figure 13 shows the pipeline stages for the chosen STAP algorithm. The chain consists of six pipeline stages, namely, video-to-I/Q conversion, array calibration, pulse compression, Doppler processing, weights computation and, finally, weights application. Each pipeline stage is briefly described below. For further details, see Cain et al. [17].

To support the STAP algorithm, a datacube corresponding to $L$ channels, $C_{p}$ pulses, and $N_{d}$ samples (ranges) must be processed each integration interval, see Figure 14.

- Video-to-I/Q-conversion

In this first stage, the digital data must be demodulated to baseband, lowpass filtered, and decimated to a lower sample rate. Demodulation to baseband is achieved by multiplying the data by coefficients that translate the signal to baseband. The samples are then processed by a lowpass filter to remove aliased frequency components and finally decimated to achieve the desired data rate. In this stage, the datacube is distributed among the processing elements (PEs) channel-wise, and each PE operates on data samples from each channel and each pulse independently.

\section{- Array calibration}

Array calibration is essential to maintain high antenna gain in the direction of the desired signal. It helps prevent the adaptive processing from nulling signals located in the main lobe of the antenna. Calibration can be achieved by applying an FIR filter to the data with filter

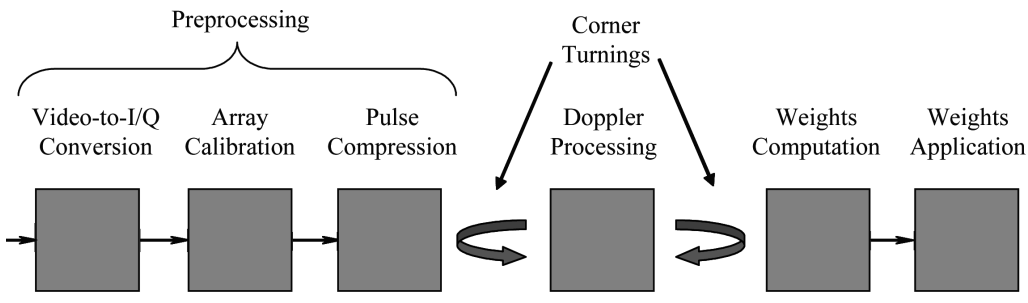

Algorithmic

Pipeline Stage

Figure 13: The algorithmic pipeline stages in the airborne STAP radar system. 


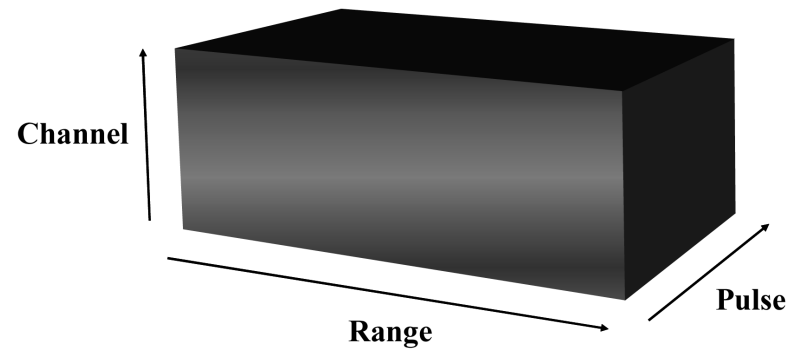

Figure 14: The three-dimensional datacube that must be processed every integration interval.

coefficients designed to equalize the antenna response. The PEs work with the output data from the previous stage and process each channel and pulse independently. Therefore, no data need to be redistributed.

- Pulse compression

To achieve high signal energy and improved detection performance, pulse compression is applied to the pulses. Pulse compression is achieved by applying an FIR filter to the data with filter coefficients matched to the received signal waveform. As in the two previous stages, each PE operates on data samples from each channel and each pulse independently. Therefore, no data need to be redistributed.

- Doppler processing

Doppler processing is a key component in all Dopplerfactored STAP algorithms. In this stage, multiple pulses are processed to separate signals based upon their Doppler frequency. Doppler processing is implemented by applying a discrete Fourier transform across multiple pulses of the preprocessed data for a given range and cell. Hence, we need to perform a corner turning before we start to compute in this stage. This is illustrated in Figure 13.

- Weights computation

In this stage, adaptive weights are calculated from the data received from multiple channels to control the spatial response of the system. The algorithm computes a set of adaptive weights, using a data domain approach that involves a matrix factorization called QR decomposition. Each PE in this stage needs data from all channels and multiple range samples but only from one pulse at a time (see Figure 15 and the following text). This means, again, that we need to perform a corner turning before this stage.

- Weights application

In this final stage, the whole concept of STAP comes into play. The weights that have been calculated in the previous stage are applied in the system to allow the algorithm to adjust its temporal and spatial response in order to null clutter returns and interference and to adapt to changes in the signal environment. No redistribution is needed before this stage.

Table 1 shows the computational load in each pipeline stage. The load is measured in floating point operations per integration interval (INTI) (and not per second). Note that all floating point calculations are derived from equations in Cain et al. [17]. Note also that the array calibration and the pulse compression stages are combined in Table 1.

Clearly, the most difficult stage to calculate is the weights computation (a factor of 100 times more calculations than the other stages).

\subsection{Hardware architecture analysis}

Let us assume for a brief moment that we only have one processor with its own memory. If we perform all calculations with one processor, we have to perform $5.17^{*} 10^{10}$ floating point operations during one INTI. This corresponds to a sustained performance of more than 1.6 TFLOPS (Tera floating point operations per second), and this is too high for a single processor. As a consequence, we must reduce the per-processor load by using several processors and by using as much time as possible without violating the latency requirement. The extended working time is achieved by pipelining some computational parts in the chain. In addition,

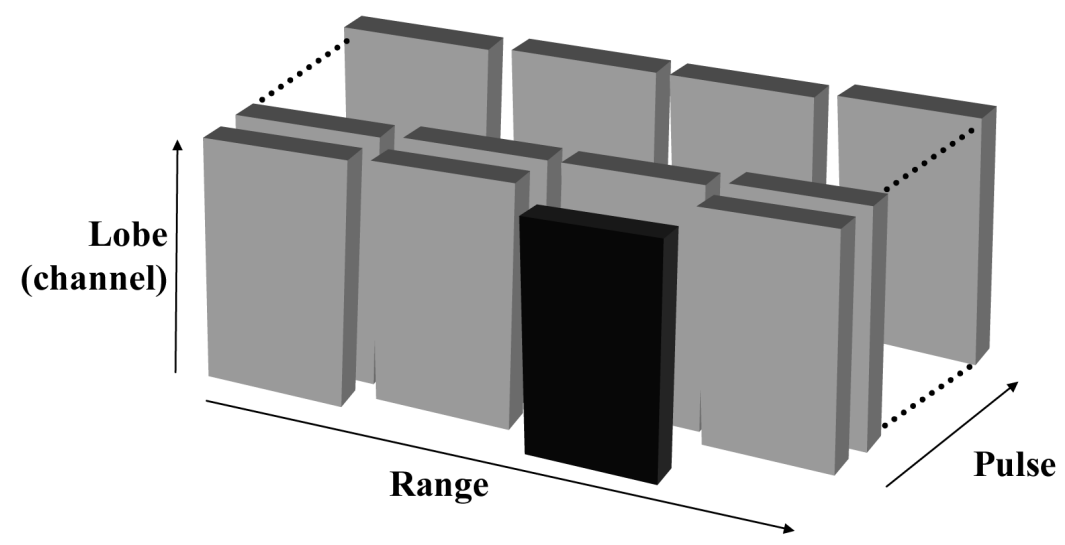

Figure 15: Distribution of QR decompositions in the datacube. The dark block corresponds to a matrix used in one $Q R$ decomposition. 


\begin{tabular}{|l|c|}
\hline Pipeline stage & $\begin{array}{c}\text { Floating-point } \\
\text { operations per INTI }\end{array}$ \\
\hline Video-to-I/Q-conversion & $4.56^{*} 10^{8}$ \\
Array cal. and pulse comp. & $4.51^{*} 10^{8}$ \\
Doppler processing & $1.28^{*} 10^{8}$ \\
Weights computation & $5.05^{*} 10^{10}$ \\
Weights calculation & $1.57^{*} 10^{8}$ \\
\hline
\end{tabular}

Table 1: Computational load in each pipeline stage in the airborne radar system (measured in floating point operations per integration interval).

when many processors are used, the time spent in interprocessor communication will be noticeable and must be included in the calculations.

Since the weights computation (WC) stage is the most critical from a computational point of view, we will start by analyzing that part. In the WC stage, QR decompositions dominate the computational complexity [17]. (A QR decomposition is a numerically stable method for triangularizing matrices [16].) The total number of QR decompositions to be computed in the entire datacube depends on the chosen algorithm. In this case study, one QR decomposition is performed on a matrix covering all channels and one-fourth of all range samples in one pulse, see Figure 15. Furthermore, this partition requires that the datacube is redistributed from Doppler to range oriented view, i.e. a corner turn must be performed in either the end of the Doppler processing (DP) stage or at the beginning of the WC stage. An obvious choice is to perform the corner turn in the stage with the lowest computational load. We thus choose to perform the corner turn in the DP stage. Also, to prevent extremely high inter-processor communication, we avoid partitioning a single $\mathrm{QR}$ decomposition over several processors. This means that $256\left(4 C_{p}\right)$ is the maximum number of processors that can be used at the same time to calculate the weights. However, it is actually possible to reduce the per-processor load even more if we use the system scalability and divide the computational work on two alternating working chains, see Figure 16. In this figure, every other datacube (with an odd number) to be processed is sent via arrow a) to the light colored group of processors. Similarly, the even number datacubes are sent via arrow b) to the dark colored group of processors. (Note that both working chains consist of 256 processors each, i.e. sixteen $5 \mathrm{D}$ hypercubes in total).

The idea underlying the approach of dividing the computational work is that the two working chains can be overlapped, see Figure 17. Using this strategy, the time to process a single datacube is doubled. As a consequence, the per-processor work is reduced to half. Finally, if we combine the weights computation and the weights application stages, we need to perform $5.07 * 10^{10}$ floating point operations on 256 processors during a time interval of $2 \tau$, i.e. we achieve a sustained per-processor floating point performance of 3.07 GFLOPS. This is very close to 3 GFLOPS and can therefore be considered acceptable.

In the rest of the computational stages, i.e. the videoto-I/Q conversion, array calibration, pulse compression and the Doppler processing stage, we must perform altogether a total of $1.03 * 10^{9}$ Flops during one INTI (the remaining time of the maximum latency), minus the time it takes to perform two corner turns, see Figure 13, and minus the time it takes to distribute data to all processors in the weights computation stage.

To be able to calculate the corner turn time, we must know the size of the datacube. The total number of samples used in every integration interval in the algorithm is $L N_{d} C_{p}$. Since every sample is complex and the real and imaginary parts are both $32 \mathrm{bit}$, the total size $\left(D_{\text {size }}\right)$ of the datacube is $\approx 252 \mathrm{Mbit}$. As a result, it will take $t_{C T}=1.47 \mathrm{~ms}$ to perform a corner turn on a $6 \mathrm{D}$ hypercube with 64 processors $(P=64)$ and $0.86 \mathrm{~ms}$ on a $7 \mathrm{D}$ hypercube with 128 processors, according to Expression 1 and the system parameters given above.

Next, we have to calculate the time it takes to distribute the data to the correct cluster of $5 \mathrm{D}$ hypercubes in

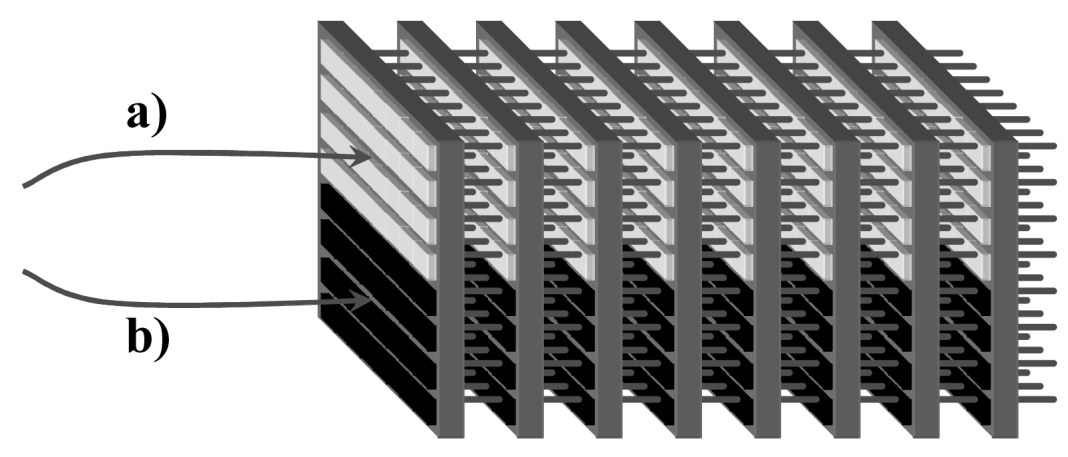

$16 * 5 D$

Figure 16: Two alternating working chains in the weights computation stage extend the working time and reduce the per-processor load. 


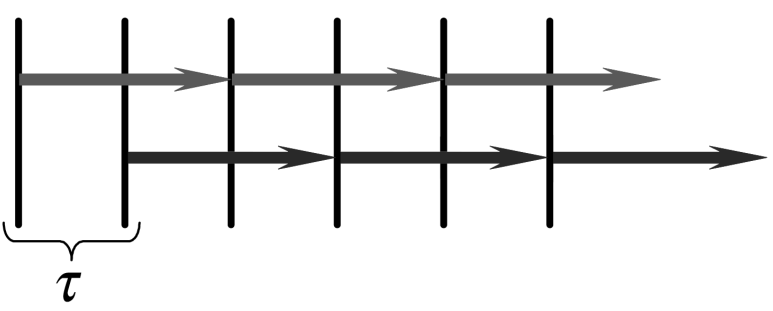

Figure 17: In every integration interval, $\tau$, a new datacube must be processed. If two working sets overlap their calculations, the computational load can be spread over twice as many processors.

the weights calculation stage, i.e. among path a) or b) in Figure 16. However, before that, we have to determine the time it takes to gather the data from a $6 \mathrm{D}$ to a $5 \mathrm{D}$ hypercube. This time can be calculated using Expression 1. However, some changes are needed since we only move data in one direction in one dimension. Therefore, we reduce the number of dimensions from $\log (P)$ to 1 . In addition, we change the number of active receivers from $P$ to $P / 2$. The time it takes to gather data from $P$ to $P / 2$ processors is then:

$$
\frac{\frac{1}{2} D_{\text {size }}}{\frac{P}{2} R_{\text {link, eff }}}
$$

seconds.

If we start from a 6D hypercube, we only have to gather data once. However, if we start from a $7 \mathrm{D}$ hypercube, we must first add the time it takes to gather data from a $7 \mathrm{D}$ to a $6 \mathrm{D}$ hypercube. Finally, we have to move all data to the first $5 \mathrm{D}$ hypercube in the CFAR chain, which in turn must move $7 / 8$ to the next $5 \mathrm{D}$ hypercube etc. This data movement can be pipelined, however, i.e. as soon as the first hypercube receives data, it immediately starts forwarding this information to the next cube etc. Therefore, this time only includes the first movement of $D_{\text {size }}$ data over $P$ data channels to the first $5 \mathrm{D}$ hypercube.

As a result, the total time to distribute data to all $5 \mathrm{D}$ hypercubes from a 6D hypercube or a $7 \mathrm{D}$ hypercube is $t_{D}=1.47 \mathrm{~ms}$ and $1.72 \mathrm{~ms}$, respectively. The time left to calculate $1.03 * 10^{9}$ Flops in a $6 \mathrm{D}$ hypercube is thus $27.84 \mathrm{~ms}\left(\tau-2 t_{C T}-t_{D}\right)$, i.e. a sustained per-processor floating point performance of 578 MFLOPS. This is well below the per-processor load needed in the weights computation stage. As a result, using a 7D hypercube in this part of the chain is not necessary. (The per-processor load using a $7 \mathrm{D}$ hypercube is 279 MFLOPS.)

The final airborne system therefore consists of nine pipelined optical substrates, i.e. 576 processors, see Figure 18. The operation will be as follows:

1. Preprocessing, Doppler processing and two corner turns are performed on the same 6D hypercube, colored green in Figure 18.

2. If the datacube has an odd number, fold it and distribute it to the upper cluster of eight 5D hypercubes, colored white in Figure 18. If the datacube has an even number, fold it and distribute it to the other cluster of 5D hypercubes, colored black in Figure 18. This folding and distribution is carried out in the same time interval as step 1.

3. Weights computation and application are both performed on the same working cluster of eight $5 \mathrm{D}$ hypercubes and during a time period equal to 2 INTIs.

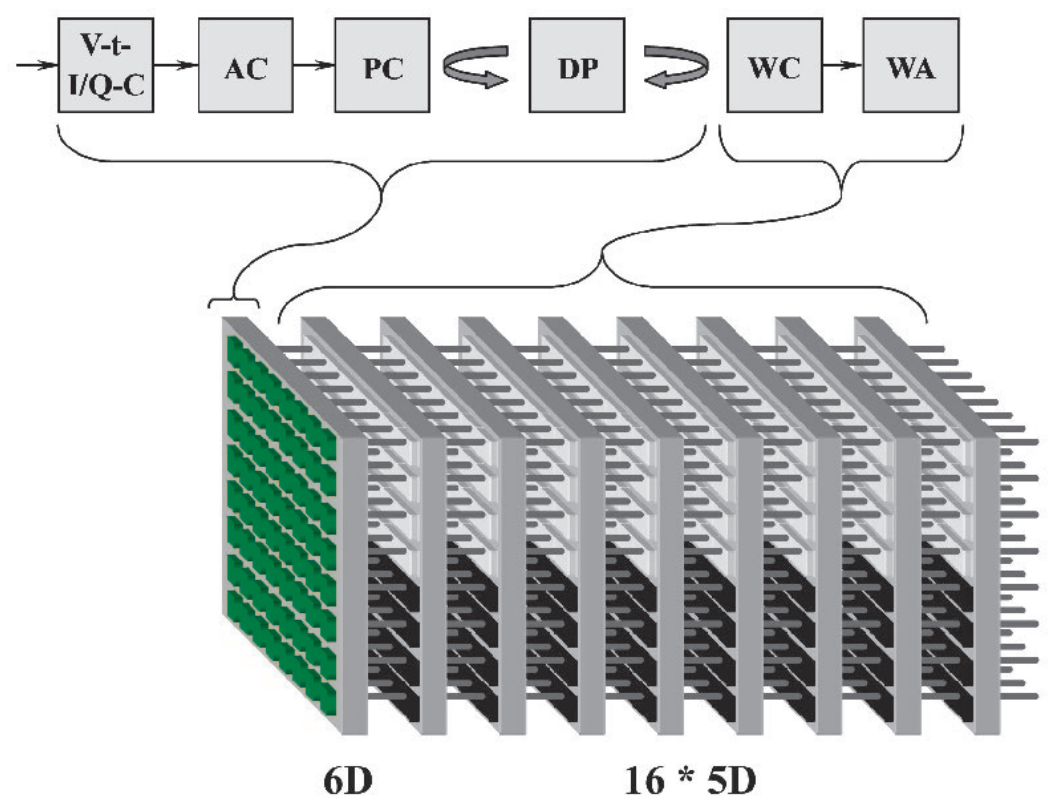

Figure 18: Final airborne radar signal processing system, one 6D hypercube and sixteen ${ }_{5}$ D hypercubes, i.e. 576 processors. 


\section{Conclusions}

This paper has presented a powerful system suitable for embedded signal processing. Several computational modules capable of working independently and sending data simultaneously are massively interconnected to meet high throughput demands. The hypercube topology forms the interconnection network within a computational module. Free-space optical interconnects and planar packaging technology make it possible to merge these multi-dimensional hypercubes into optical planes. Beam splitters reduce the number of transmitters and thus also the hardware cost.

An airborne STAP radar application challenged the architecture in terms of computational load and interprocessor data transfer. With a sustained per-processor performance of slightly more than 3 GFLOPS, a total of 576 processors and a bisection bandwidth of more than $1 \mathrm{Tbit} / \mathrm{s}$, the system was capable of meeting all requirements.

It can be noted that solutions that are non-optimal, in the sense that there is no overlap between computation and communication, put higher demands on the architecture. However, not putting as great an effort into optimizing overlap simplifies the software development, thus increasing engineering efficiency. On the other hand, if more suitable mappings of the algorithms are developed (at the expense of higher complexity), more powerful systems can be built using this new hardware architecture.

\section{Acknowledgment}

This work is financed by Ericsson Microwave Systems within the Intelligent Antenna Systems Project.

\section{References}

[1] W. Liu and V. K. Prasanna, "Utilizing the power of high performance computing," IEEE Signal Processing Magazine, vol. 15, no. 5, Sept. 1998, pp. 85100.

[2] D. P. Bertsekas and J. N. Tsitsiklis, Parallel and Distributed Computation: Numerical Methods, PrenticeHall, inc., Englewood Cliffs, NJ, USA, 1989.

[3] M. Jonsson, High Performance Fiber-Optic Interconnection Networks for Real-Time Computing Systems, Doctoral Thesis, Department of Computer Engineering, Chalmers University of Technology, Göteborg, Sweden, Nov. 1999, ISBN 91-7197-852-6. Thesis available at: http:/ /www.hh.se/stafflmagnusj/

[4] K. Teitelbaum, "Crossbar tree networks for embedded signal processing applications," Proceedings of Massively Parallel Processing using Optical Interconnections, MPPOI'98, Las Vegas, NV, USA, June 15-17, 1998, pp. 200-207.

[5] H. Forsberg, "Parallel computer architectures using optical interconnects," Licentiate Thesis, Technical Report no. 379L, Department of Computer
Engineering, Chalmers University of Technology, Göteborg, Sweden, March 2001.

[6] M. D. Grammatikakis, D. F. Hsu, and M. Kraetzl, Parallel System Interconnections and Communications, CRC Press, Boca Raton, Florida, USA, 2001.

[7] H. M. Ozaktas, "Fundamentals of optical interconnections - a review," Proceedings of Massively Parallel Processing using Optical Interconnections, MPPOI'97, Montreal, Canada, June 22-24, 1997, pp. 184-189.

[8] H. M. Ozaktas, "Toward an optimal foundation architecture for optoelectronic computing. Part I. Regularly interconnected device planes," Applied Optics, vol. 36, no. 23, Aug. 10, 1997, pp. 5682-5696.

[9] H. M. Ozaktas, " Toward an optimal foundation architecture for optoelectronic computing. Part II. Physical construction and application platforms," Applied Optics, vol. 36, no. 23, Aug. 10, 1997, pp. 5697-5705.

[10] J. Jahns, "Planar packaging of free-space optical interconnections," Proceedings of the IEEE, vol. 82, no. 11, Nov. 1994, pp. 1623-1631.

[11] J. Jahns, "Integrated free-space optical interconnects for chip-to-chip communications," Proceedings of Massively Parallel Processing using Optical Interconnections, MPPOI'98, Las Vegas, NV, USA, June 15-17, 1998, pp. 20-23.

[12] D. E. Culler, J.P. Singh, with A. Gupta, Parallel Computer Architecture: A Hardware/Software Approach, Morgan Kaufmann Publishers, Inc., San Francisco, CA, USA, 1999.

[13] I. Foster, Designing and Building Parallel Programs: Concepts and Tools for Parallel Software Engineering, Addison Wesley Publishing Company, Inc., Reading, MA, USA, 1995.

[14] R. Klemm, "Introduction to space-time adaptive processing," The Institution of Electrical Engineers (IEE), Savoy Place, London WC2R OBL, UK, 1998.

[15] M. Jonsson, "Fiber-optic interconnection networks for signal processing applications," 4th International Workshop on Embedded HPC Systems and Applications (EHPC'99) held in conjunction with the 13th International Parallel Processing Symposium \& 10th Symposium on Parallel and Distributed Processing (IPPS/SPDP '99), San Juan, Puerto Rico, Apr. 16, 1999. Published in Lecture Notes in Computer Science, vol. 1586, Springer Verlag, 1999, pp. 1374-1385, ISBN 3-540-65831-9.

[16] M. Taveniku and A. Åhlander, "Instruction statistics in array signal processing," Research Report, Centre for Computer Systems Architecture, Halmstad University, Sweden, 1997.

[17] K. C. Cain, J. A. Torres, and R. T. Williams, "RT_STAP: Real-time space-time adaptive processing benchmark," MITRE Technical Report, The MITRE Corporation, Center for Air Force C3 Systems, Bedford, MA, USA, 1997. 
[18] A. Louri, B. Weech, and C. Neocleous, "A spanning multichannel linked hypercube: a gradually scalable optical interconnection network for massively parallel computing," IEEE Transactions on Parallel and Distributed Systems, vol. 9, no. 5, May 1998, pp. 497-512.

[19] F. Zane, P. Marchand, R. Paturi, and S. Esener, "Scalable network architectures using the optical transpose interconnection system (OTIS)," Journal of Parallel and Distributed Computing, 60:5, 2000, pp. 521-538.

[20] T. M. Pinkston and J. W. Goodman, "Design of an optical reconfigurable shared-bus-hypercube interconnect," Applied Optics, vol. 33, no. 8, 10 March 1994, pp. 1434-1443.

\section{Håkan Forsberg}

\section{rapid@ce.chalmers.se}

Hakan Forsberg received his M.S. degree in computer science and engineering from Linköping University, Sweden, in 1997, and Licentiate of Technology degree in computer engineering from Chalmers University of Technology, Gothenburg, Sweden, in 2001. From 1996 to 1998,

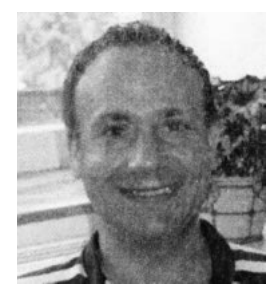

Mr. Forsberg was an electronic design and research engineer at Saab Avionics AB. Currently, he is with the Department of Computer Engineering at Chalmers University of Technology. His Ph.D. Studies are concerned with parallel computer architectures using optical interconnects.

\section{Magnus Jonsson} magnus.jonsson@ide.hh.se

Magnus Jonsson received his B.S. and M.S. degrees in computer engineering from Halmstad University, Sweden, in 1993 and 1994, respectively. He then obtained the Licentiate of Technology and Ph.D. degrees in computer engineering

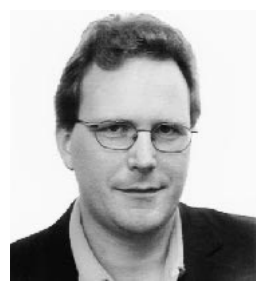

from Chalmers University of Technology, Gothenburg, Sweden, in
1997 and 1999, respectively. From 1998 to March 2003, he was Associate Professor of Data Communication at Halmstad University (acting between 1998 and 2000). Since April 2003, he has been Professor of Real-Time Computer Systems at Halmstad University. He has published about 35 scientific papers, most of them in the area of optical communication and real-time communication. Most of his research is targeted for embedded, industrial and parallel and distributed computing and communication systems. Dr. Jonsson has served on the program committees of International Workshop on Optical Networks, IEEE International Workshop on Factory Communication Systems, International Conference on Computer Science and Informatics, and International Workshop on Embedded/Distributed HPC Systems and Applications.

\section{Bertil Svensson}

bertil.svensson@ide.hh.se

Bertil Svensson received his M.Sc. in Electrical Engineering from the University of Lund, Sweden, in 1970 and his Ph.D. in Computer Engineering from the same University in 1983. He was an Assistant Professor and Vice President of Halmstad

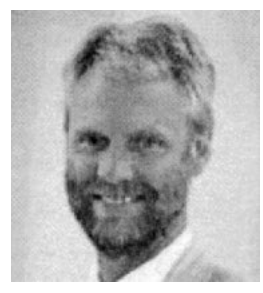
University, Halmstad, Sweden, before he, in 1991, was appointed professor of Computer Systems Engineering at Chalmers University of Technology in Gothenburg, Sweden. From 1998 he is Professor of Computer Systems Engineering at Halmstad University and Chalmers. He is also Dean of the School of Information Science, Computer and Electrical Engineering at Halmstad University.

Prof. Svensson is research leader of the Laboratory for Computing and Communication at Halmstad University. His research interests include massively parallel architectures, applicationoriented architectures for embedded systems, reconfigurable architectures, and optically interconnected parallel systems. 